\title{
Reabilitação oral com implantes em paciente transplantado imunossuprimido: relato de caso clínico
}

\author{
Oral rehabilitation with implants in immunosuppressed transplanted patient: a case report \\ Reabilitación oral con implantes en paciente trasplante inmunosupprimido: caso clínico \\ Fabianni Magalhães Apolonio ${ }^{1}$, Darius Nakai do Rego Barros ${ }^{1}$, Rodrigo Marochio Pavane ${ }^{1}$, Julia \\ Lima $^{2}$, Mário Ferreira Filho ${ }^{1 *}$.
}

\section{RESUMO}

Objetivo: Descrever um caso clínico de reabilitação oral em paciente transplantado imunossuprimido. Detalhamentos de caso: Paciente do gênero masculino, 58 anos de idade, transplantado hepático e hipertenso, buscou tratamento reabilitador com a queixa de insatisfação estética e funcional da maxila e mandíbula. O paciente fazia uso do imunossupressor de manutenção tacrolimus, e possuía baixas taxas de dosagem da medicação e quantidade de leucócitos normal. Após avaliação clínico/radiográfica, foram planejados 6 implantes em maxila, para confecção de prótese protocolo superior e 3 implantes em mandíbula, para reabilitação de espaços edêntulos intercalados. Com o objetivo de reduzir as complicações cirúrgicas, aumentar a previsibilidade do caso e dar maior segurança ao paciente, optou-se pela realização de cirurgia guiada com implante cônico. Considerações finais: É possível considerar através deste trabalho que um planejamento seguro, envolvendo uma boa anamnese, análise de exames laboratoriais, medicação préoperatória e planejamento cirúrgico-reabilitador adequado são a chave para um tratamento de sucesso.

Palavras-chave: Implante dentário, Reabilitação bucal, Hospedeiro imunocomprometido, Qualidade de vida.

\begin{abstract}
Objective: To describe a clinical case of oral rehabilitation in an immunosuppressed transplant patient. Case report: Male patient, 58 years old, liver transplanted and hypertensive, sought rehabilitation treatment complaining of aesthetic and functional dissatisfaction of the maxilla and mandible. The patient was using the maintenance immunosuppressant tacrolimus and had low drug dosage rates and normal white blood cell count. After clinical and radiographic evaluation, 6 implants in the maxilla were planned for making a superior protocol prosthesis and 3 implants in the mandible for the rehabilitation of intercalated edentulous spaces. Con el fin de reducir las complicaciones quirúrgicas, aumentar la predictibilidad del caso y brindar mayor seguridad al paciente, optamos por realizar una cirugía guiada con un implante cónico. Final considerations: It is possible to consider through this work that a safe planning, involving a good anamnesis, analysis of laboratory tests, preoperative medication and adequate surgical-rehabilitation planning are the key to a successful treatment.
\end{abstract}

Key words: Dental implantation, Mouth rehabilitation, Immunocompromised host, Quality of life.

\section{RESUMEN}

Objetivo: Describir un caso clínico de rehabilitación oral en un paciente trasplantado inmunodeprimido. Datos del caso: Paciente masculino de 58 años, trasplantado de hígado e hipertenso, que solicitó tratamiento rehabilitador por insatisfacción estética y funcional de maxilar y mandíbula. El paciente estaba usando el

1 CEPROEDUCAR, Manaus - AM. *E-mail: dr.mfilho@gmail.com

2 Centro Universitário do Norte (UNINORTE), Manaus - AM.

SUBMETIDO EM: 7/2021 
inmunosupresor de mantenimiento tacrolimus, tenía bajas tasas de dosificación del fármaco y recuento normal de glóbulos blancos. Después de la evaluación clínica radiográfica, se planificaron 6 implantes en el maxilar para realizar una prótesis de protocolo superior y 3 implantes en la mandíbula para la rehabilitación de espacios edéntulos intercalados. Para reducir las complicaciones quirúrgicas y brindar mayor seguridad al paciente, optamos por realizar una cirugía guiada con un implante cónico. Consideraciones finales: Es posible considerar a través de este trabajo que una planificación segura, que incluya una buena anamnesis, análisis de pruebas de laboratorio, medicación preoperatoria y una adecuada planificación quirúrgicarehabilitadora son la clave para un tratamiento exitoso.

Palabras clave: Implantación dental, Rehabilitación bucal, Huésped inmunocomprometido, Calidad de vida.

\section{INTRODUÇÃO}

O uso de implantes para a reabilitação oral de pacientes tem se mostrado uma excelente alternativa que melhora significativamente a estética e a função oral (mastigação, deglutição e fala) do paciente. Atualmente o tratamento com implantes necessita de cirurgia menos invasiva com menos risco de complicações graves. Além disso, mais estudos mostram que o tratamento com implantes dentários em muitas doenças é viável, seja com ou sem precauções específicas. É uma terapia que proporciona vantagens biológicas e funcionais em relação às próteses removíveis, sendo o tratamento que mais se assemelha às funções dos dentes naturais hígidos. Os implantes são um tratamento previsível com taxas de sucesso e sobrevivência em torno de 95\% (BUSER D, et al., 2017).

O transplante de órgãos sólidos no Brasil cresceu consideravelmente durante os anos de 2001 a 2017 , transformando o Brasil em referência mundial neste setor. $O$ país já representa o segundo lugar mundial em número absoluto de transplantes de fígado e rins. Cerca de $96 \%$ dos procedimentos realizados em 2018 foram financiados pelo Sistema Único de Saúde (SUS) (MINISTÉRIO DA SAÚDE, 2018).

Esse crescimento pode ser atribuído ao aumento do número e distribuição dos Centros de Transplantes no Brasil. De acordo com a Associação Brasileira de Transplante de Orgãos (ABTO), no ano de 2019, foram realizados 24.130 transplantes de órgãos (ABTO, 2020).

O aumento no número de transplantados e a elevação do índice de sucesso de transplantes de órgãos sólidos, tem aumentado a expectativa de vida dos pacientes e ampliado a procura por reabilitação oral e melhora da qualidade de vida (GU L e YU Y-C, 2011).

Para garantir a longevidade da função no órgão recebido, os pacientes transplantados devem ser submetidos a terapia com agentes imunossupressores de manutenção que podem provocar efeitos colaterais importantes para o planejamento cirúrgico, como: complicações metabólicas, leucopenia, anemia e trombocitopenia. Além disso, a medicação imunossupressora torna os pacientes mais suscetíveis a certas patologias, como as infecções (PAREDES V, et al., 2017).

Uma vez recuperados da cirurgia de transplante, a reabilitação com implantes deve ser considerada uma alternativa viável para estes pacientes (VISSINK A, et al., 2018). No entanto, devido a pouca quantidade de artigos relacionando o uso crônico de imunossupressores e o sucesso dos implantes, alguns profissionais ainda possuem receio de intervir cirurgicamente em pacientes transplantados (GU L e YU Y-C, 2011).

O uso de técnicas cirúrgicas mais avançadas, com maior previsibilidade e menor comorbidade podem ser uma excelente alternativa para pacientes transplantados. A confecção de guias estáticos, a partir de dados tridimensionais (3D) obtidos a partir de tomografia computadorizada cone beam, escaneamento intraoral, planejamento virtual de implantes e fabricação de guias por computador (CAD/CAM), auxiliam a cirurgia e trazem maior segurança cirúrgica. O guia cirúrgico fabricado pode ser suportado por dente, mucosa ou osso. As cirurgias guiadas promovem cirurgias sem a abertura de retalho e descolamento do periósteo, reduzindo a agressão aos tecidos e a contaminação das áreas cirúrgicas adjacentes, possibilitando uma cicatrização mais rápida para o paciente. Além disso, a técnica reduz o tempo de cirurgia e as complicações pós- 
operatórias como dor e edema, gerando um pós-cirúrgico mais confortável para o paciente (AL YAFI F, et al., 2019).

O objetivo do presente estudo foi relatar um caso clínico de reabilitação oral através da instalação de implantes com cirurgia guiada em maxila e mandíbula em paciente transplantado hepático imunossuprimido.

\section{DETALHAMENTO DO CASO}

Paciente do gênero masculino, 58 anos de idade, compareceu à Clínica da Especialização em Implantodontia com a queixa de insatisfação estética e funcional da maxila e mandíbula. O paciente era usuário de prótese parcial removível à grampos superior e possuía algumas ausências dentárias inferiores. Relatou descontentamento estético e funcional com próteses removíveis e o anseio em usar próteses fixas sobre implante. O paciente já havia procurado tratamento com outros profissionais que haviam contraindicado a cirurgia de implantes devido a imunossupressão, mas desejava ouvir uma segunda opinião. A Figura 1A mostra a foto inicial de perfil do paciente (Figura 1A). As Figura 1B e Figura 1C mostram as fotos iniciais do paciente com e sem a Prótese Parcial Removível anterior (Figura 1B e Figura 1C).

Figura 1 - Fotos iniciais do sorriso do paciente.

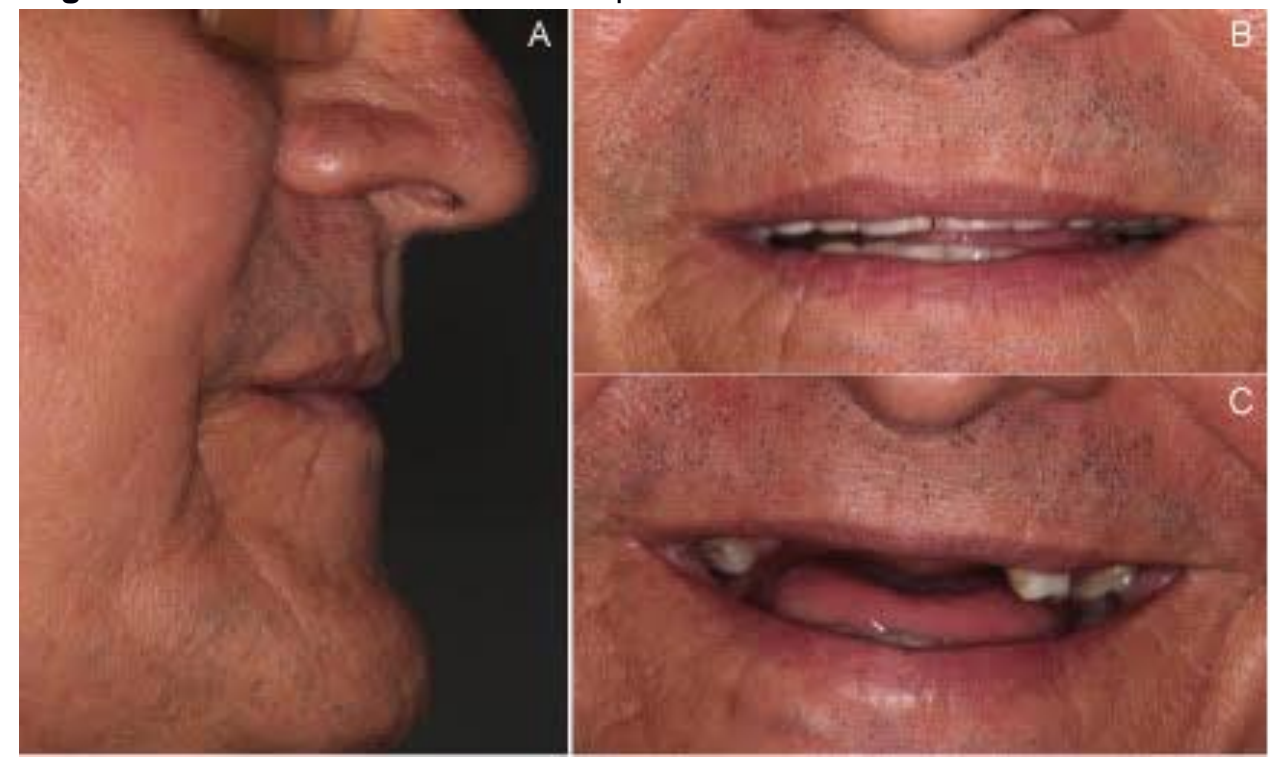

Fonte: Apolonio FM, et al., 2021.]

Durante a anamnese, o paciente relatou ter realizado transplante hepático à 6 anos devido a cirrose hepática. Para manutenção do órgão sólido doado, o paciente fazia uso contínuo do imunossupressor Tacrolimus. O paciente relatou também ser hipertenso controlado através do uso de Losartana e usar Metformina para prevenir o desenvolvimento de diabetes. $O$ paciente apresentou exames laboratoriais recentes de contagem de leucócitos, glicose em jejum, função hepática, função renal e a concentração sanguínea do fármaco imunossupressor sem alterações. O Paciente possuía boa saúde periodontal e higiene bucal satisfatória, sem lesões bucais ou periapicais dos dentes remanescentes.

Por se tratar de um paciente hipertenso e com uso crônico de imunossupressor, optou-se por realizar uma cirurgia guiada com corte "flapless" a fim de reduzir a morbidade cirúrgica, tempo de cirurgia e complicações pós-operatórias. Portanto, foram realizadas tomografia computadorizada para planejamento de implantes e escaneamento intra-oral para realização de planejamento virtual. Obtidas as imagens tridimensionais, simulou-se, por meio do software Blue Sky Plan, a distribuição de: 1) 6 implantes na maxila, com exodontia do dente 23 e planejamento reabilitador através de prótese protocolo e; 2) 3 implantes na mandíbula nas áreas dos dentes 36, 45 e 46 (Figura 2). 
Figura 2 - Planejamento radiográfico do caso clínico.

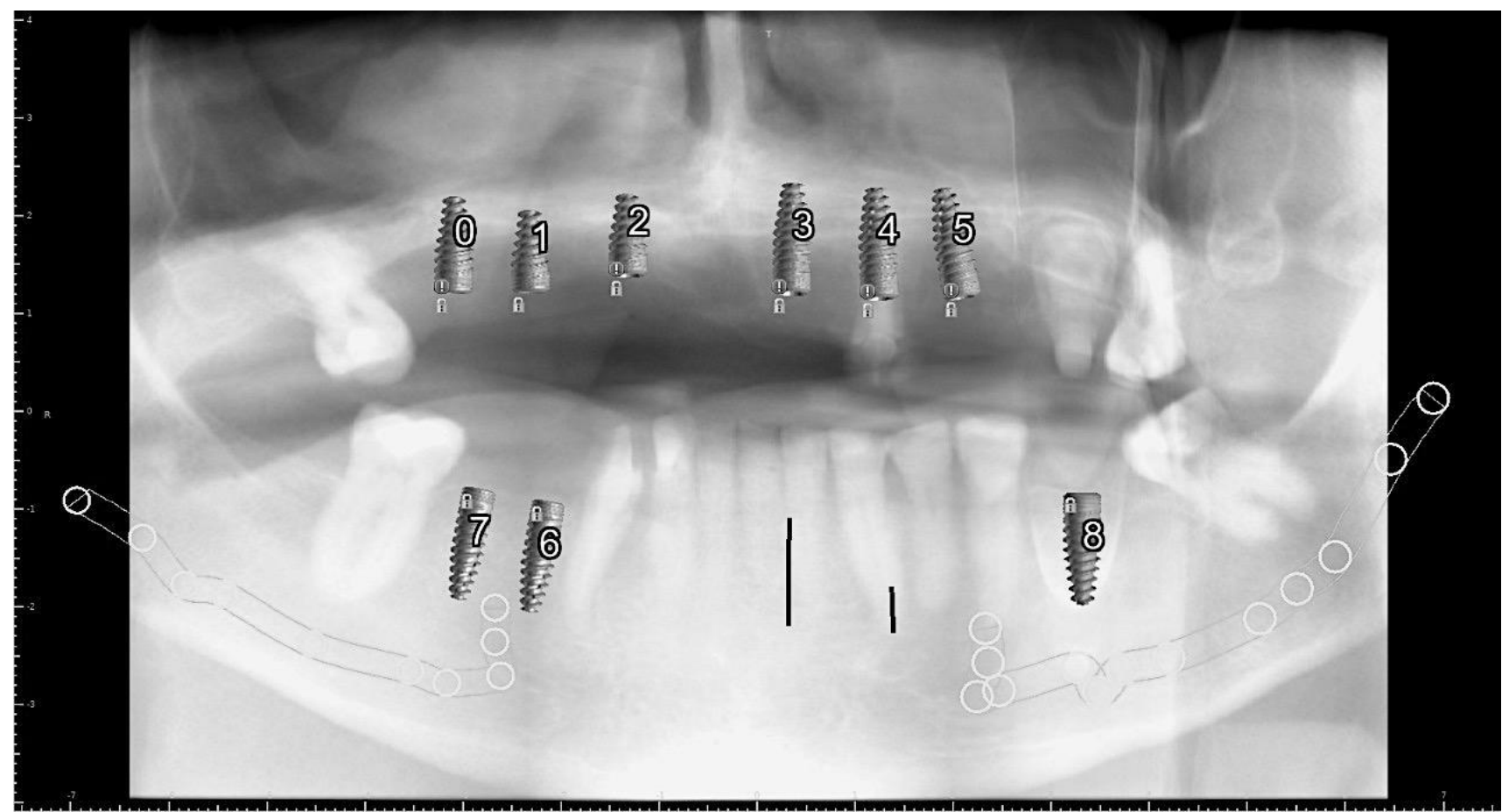

Fonte: Apolonio FM, et al., 2021.

Os dentes 17 e 27 foram mantidos a fim de preservar a Dimensão Vertical de Oclusão. Optou-se pela instalação de implantes do tipo cone morse cônicos com tratamento de superfície. $O$ arquivo final contendo todas as informações do planejamento deste caso foi impresso em Impressora Anycubic Photon para a confecção do guia cirúrgico (Figura 3A e Figura 3B).

Figura 3 - Guias cirúrgicas impressas.

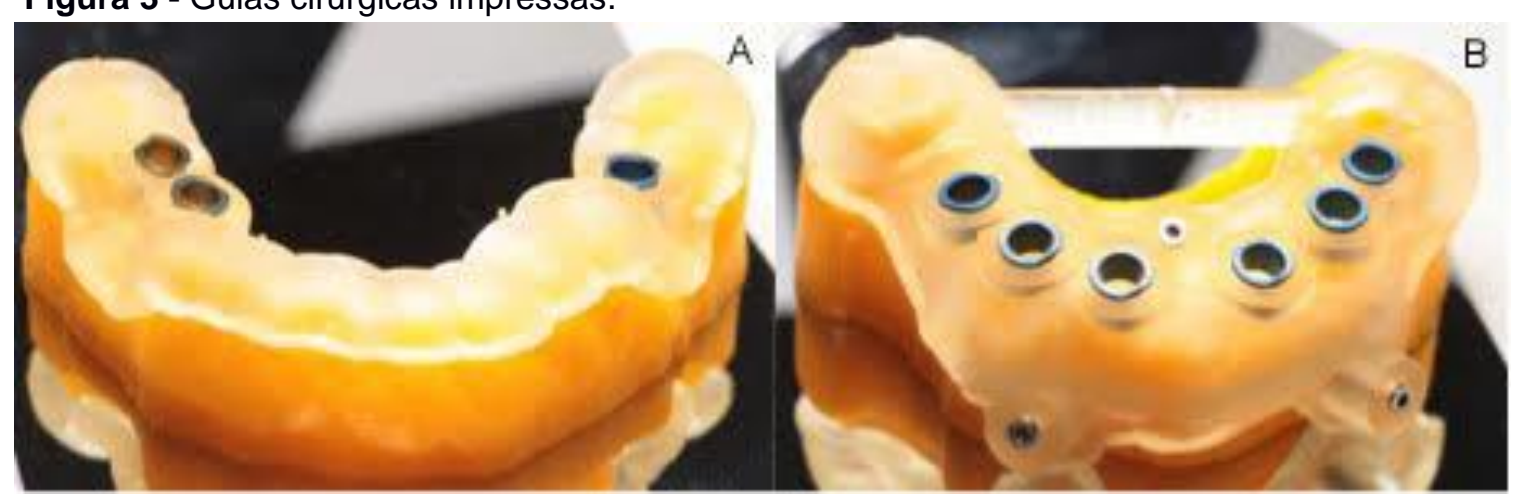

Fonte: Apolonio FM, et al., 2021.

Para controlar prevenir infecção pós-operatória, e seguir a literatura atual proposta, foi prescrito o uso de antibiótico profilático, Amoxicilina 500mg + Clavulanato de potássio $125 \mathrm{mg}$, e o analgésico Dipirona $1 \mathrm{~g}$ para controle de dor pós-cirúrgica.

A cirurgia iniciou-se com a exodontia minimamente traumática do dente 23 , com preservação de toda a parede óssea vestibular. Foi realizada a prova e a fixação do guia cirúrgico da maxila através dos pinos de fixação, anestesia local nas áreas dos implantes e os procedimentos de fresagem dos 6 implantes de acordo com o planejamento cirúrgico (Figura 4). Após a instalação dos implantes da maxila, iniciou-se a cirurgia da mandíbula com fixação do guia cirúrgico apoiado e fixado sobre os dentes remanescentes e fresagem dos 3 implantes planejados. 
Figura 4 - Fresagem dos implantes utilizando as guias cirúrgicas.

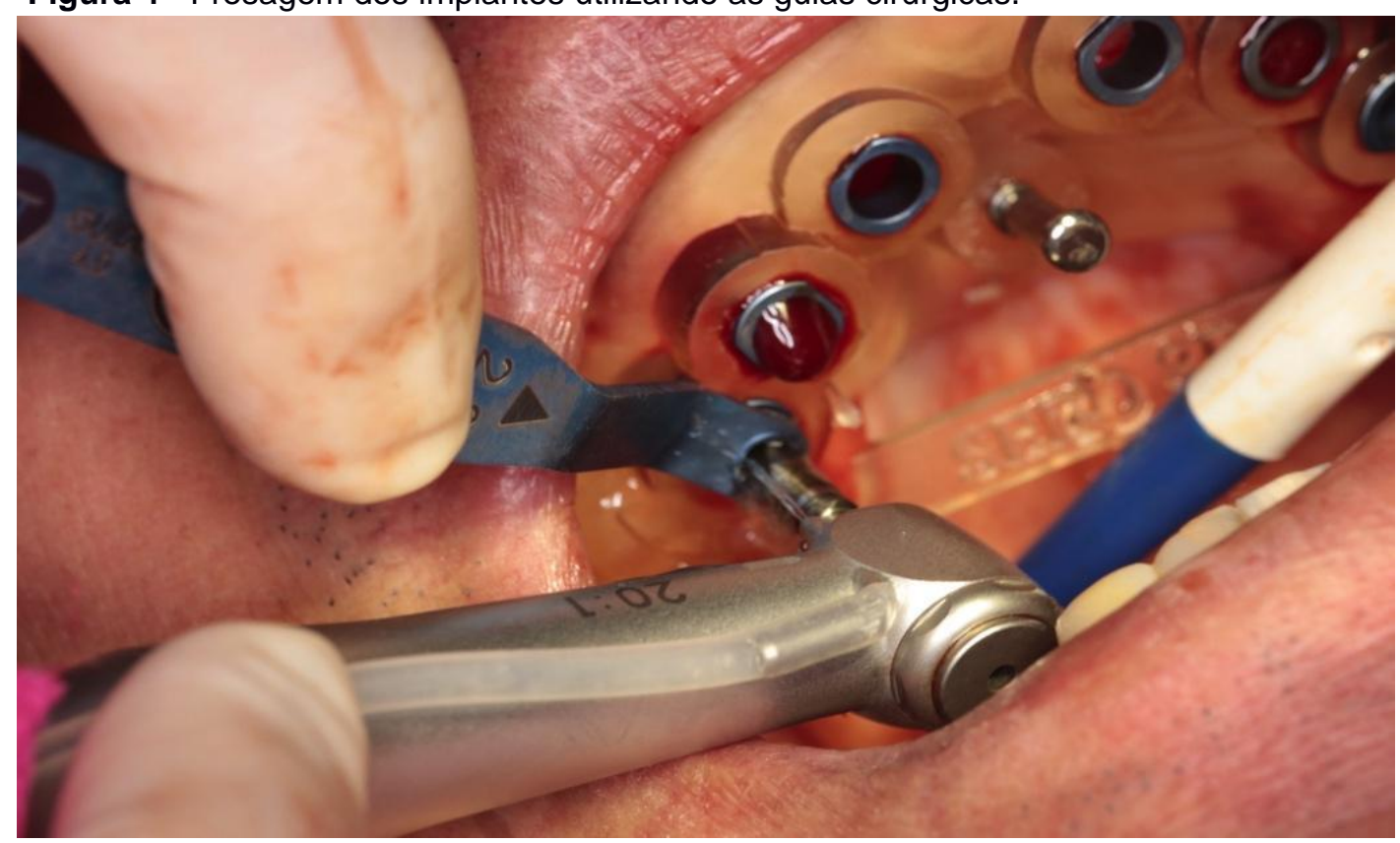

Fonte: Apolonio FM, et al., 2021.

Finalizadas as fresagens e instalação dos implantes, removeu-se o Guia Cirúrgico e pode-se observar quão conservador foi o procedimento, quão benefício aos tecidos ósseo e gengivais e por fim, quão menos agressivo foi ao paciente. O paciente retornou após 1 semana para controle pós-operatório e relatou não ter sentido dor ou edema.

\section{DISCUSSÃO}

Ao se planejar uma reabilitação oral é preciso ter certeza que a opção apresentada ao paciente é a melhor possível para o seu caso clínico. Para isso, deve ser levado em consideração as suas condições de saúde sistêmica, seus anseios em relação ao resultado e suas condições financeiras. É preciso avaliar bem todas estas condições para não gerar expectativas infundadas ou frustrações ao paciente.

De acordo com Vissink A, et al. (2018) ao indicar o uso de implantes em paciente comprometido, seis questionamentos devem ser considerados: $O$ tratamento com implante pode representar um risco para a saúde do paciente; existe alguma precaução específica para a colocação de implantes; caso exista alguma complicações no implante, estas podem ser controladas; o sistema de implantes usado e a técnica cirúrgica são cientificamente comprovadas e possuem um resultado favorável a longo prazo, tanto em relação à sobrevivência do implante e a saúde perimplantar; o acompanhamento profissional é bem organizado e protegido por um longo período de tempo; e o paciente ou cuidador é capaz de manter os cuidados bucais necessários (VISSINK A, et al., 2018).

No caso clínico relatado, o paciente ansiava por uma reabilitação fixa que melhorasse sua qualidade de vida, capacidade mastigatória e estética. $O$ tratamento mais indicado, nestes casos, é a reabilitação com implantes. Embora outros cirurgiões-dentistas tenham contraindicado a cirurgia para este paciente, sua esperança por um tratamento melhor e mais humanizado fez com que ele procurasse nossa equipe. Foi realizada uma anamnese detalhada abrangendo todos os aspectos de saúde sistêmica, uso de medicações, histórico de saúde sistêmica e bucal, com investigação sobre o tratamento realizado antes do transplante hepático e o uso de medicações usadas durante o tratamento e após o transplante de órgão.

Como qualquer outro paciente com comprometimento sistêmico e medicamentoso, é recomendado aguardar a estabilização do sistema imune antes do planejamento reabilitador com implantes (VISSINK A, et al., 2018). O paciente havia realizado cirurgia de transplante hepático há 6 anos, sendo considerado, portanto 
um paciente com período pós-transplante tardio. O paciente possuía exames laboratoriais que comprovaram a estabilidade do tratamento imunossupressor.

Os pacientes que recebem transplantes de órgãos sólidos, precisam de terapia imunossupressora de manutenção para o resto da vida. O uso contínuo destas medicações pode retardar a cicatrização de feridas, aumentar as chances de infecções oportunistas e alterar o metabolismo ósseo, pela inibição da função dos osteoblastos e da osteogênese ocasionando aumento de reabsorção óssea e osteoporose (MOLON R, et al., 2017). Estas alterações sistêmicas provocadas pelo uso dos imunossupressores, pode se tornar uma precaução específica para a colocação de implantes e induzir alguns profissionais a contraindicar a reabilitação oral com uso de implantes dentários.

A contraindicação para instalação de implantes em pacientes imunossuprimidos, na maioria dos casos, se dá por receio ou desconhecimento das alterações promovidas pela terapia imunossupressora a que estes pacientes são submetidos. A ausência de ampla literatura de suporte para planejamento de casos de pacientes imunossuprimidos corrobora com este receio (BORNSTEIN MM, et al., 2009). Foram encontrados somente dois relatos de caso clínico relacionados ao tratamento com implantes em pacientes transplantados hepáticos e dois estudos controlados prospectivos, um em pacientes com transplante de fígado e o outro em paciente submetido a transplante combinado de coração e fígado (GU L, et al., 2011; HECKMANN SM, et al., 2004; PAREDES V, et al., 2017; MONTEBUGNOLI L, et al., 2012). Nenhum destes estudos relatava o uso de cirurgia guiada e prótese protocolo em pacientes imunossuprimidos.

O paciente fazia uso do Tacrolimus (TAC), um dos principais imunossupressores de manutenção que atua inibindo a calcineurina, de modo semelhante à ciclosporina (CsA), mas com menos efeitos secundários. Ela inibe uma enzima chave para a produção de IL-2 pelas células $\mathrm{T}$, bloqueando a ativação e proliferação linfocitária. Essa droga tem mostrado aumento da reabsorção óssea dos ossos esponjosos e trabeculares (HOLT CD, 2017).

Estudo realizado em ratos, relatou um efeito negativo do TAC na osseointegração dos implantes dentários e sugeriu que esse resultado pode ser fruto da inibição das células T (ZHENG X, et al., 2017). No entanto, estudos clínicos sugerem ausência de efeito do TAC na rejeição de implantes dentários. Um estudo avaliou a estabilidade do implante dentário instalado em mandíbula de paciente transplantado. Durante oito anos, nem o implante, nem a prótese mostraram complicações (NAKAGAWA A, et al., 2014).

Um outro estudo clínico avaliou o sucesso de 11 implantes instalados em paciente 1 ano após a cirurgia de transplante e tratado com TAC para imunossupressão. Após cinco anos, não foi observado perda dos implantes. Os autores atribuíram alguns fatores ao sucesso do tratamento, são eles: o exame clinico minucioso, a prescrição de antibióticos e o procedimento minimamente invasivo do implante dentário (GU L, et al., 2011). Em 2015, o mesmo grupo realizou testes microbiológicos avaliando a microbiota subgengival da região peri-implantar de pacientes transplantados imunossuprimidos e de pacientes controle. Não foi observado diferença estatística nos testes microbiológicos, sem aumento no número de microrganismos patógenos, nem alterações na perda óssea ao redor dos implantes (MONTEBUGNOLI L, et al.,2015).

Paredes V, et al. (2017), realizaram um estudo comparando pacientes imunossuprimidos com transplante de fígado e pacientes controle submetidos a tratamento com implantes. Estes pacientes foram acompanhados por 8 anos e foram avaliados usando parâmetros clínicos, periodontais e radiográficos. Foi observado que os pacientes tratados com imunossupressores não tiveram mais complicações pós-cirurgicas e/ou dor do que os pacientes controles não imunossuprimidos. Além disso, a incidência de peri-implantite ou perda óssea nestes pacientes foi ainda menor do que a dos pacientes controle, embora essas diferenças não sejam significativamente diferentes.

Um outro estudo de metanálise, avaliando 107 implantes colocados em 39 pacientes transplantados, não encontrou correlações estatisticamente significantes entre a imunossupressão pós transplante e a falha do implante. Todos os estudos e relatos de caso avaliados descreveram uma taxa de sobrevivência do implante de $100 \%$. Foi utilizado antibiótico profilático, com moxifloxacina durante seis dias ou amoxicilina com ácido clavulânico durante cinco a seis dias (DUTTENHOEFER F, et al., 2019). 
Vários estudos mostraram que o tratamento com implantes em pacientes imunocomprometidos é viável quando as precauções adequadas são tomadas, principalmente sendo profilaxia antibiótica (ALBRECHT K, et al., 2016; KORFAGE A, et al., 2016; KOTSAKIS GA, et al., 2015). O uso de profilaxia antibiótica pode atuar inibindo infecções oportunistas que podem acometer os pacientes imunossuprimidos. Tendo em vista esses achados, interpreto como obrigatória a prescrição de antibióticos pré-cirúrgicos, quando se trata de pacientes imunossuprimidos. Para o caso clínico reportado, optados pelo protocolo com uso de Amoxicilina + Clavulanato de potássio $24 \mathrm{~h}$ antes do procedimento cirúrgico e manutenção do uso por 5 dias.

A necessidade de higiene oral também foi amplamente relatada nos estudos abordados e é extremamente importante para todos os casos cirúrgicos. Durante a avaliação clínica do paciente, não foi observado sangramento gengival, mobilidade ou placa bacteriana visível. Foi relatado durante a anamnese que as perdas dentárias foram realizadas antes da cirurgia de transplante. O paciente não possuía problemas de coordenação motora que comprometesse sua higiene oral e foi alertado sobre a sua responsabilidade em relação às condutas corretas de higiene e manutenção das próteses.

Foi realizado um planejamento virtual com confecção de guia cirúrgico para cirurgia guiada de implantes. A cirurgia guiada é uma técnica inovadora e cientificamente comprovada que apresenta grandes vantagens em relação às técnicas convencionais, tais como a não abertura de retalho com exposição de periósteo e maior previsibilidade, ocasionando um excelente pós-operatório. A utilização dos guias garantiu uma cirurgia sem retalho, mais segura, previsível, com menor tempo cirúrgico, menor quantidade de anestésico utilizado e redução de sangramento, dor e edema. Embora apresente um custo mais elevado, aparato técnico diferenciado, necessidade de aperfeiçoamento profissional, é uma técnica promissora na odontologia (AL YAFI F, et al., 2019).

Com base no apresentado, podemos observar que a colocação de implantes dentários em pacientes submetidos a transplante de órgãos e imunossupressão não é contraindicada e traz benefícios funcionais significativos. A sobrevivência do implante e a saúde peri-implantar nesses pacientes são comparáveis às de indivíduos saudáveis. No entanto, é evidente que uma análise criteriosa de parâmetros fisiológicos e de saúde bucal deve ser mandatória. A cirurgia realizada deve ser minimamente invasiva com uso de antibiótico profilático, a fim de reduzir o risco de infeção. Um alto nível de cuidado de acompanhamento deste tipo de reabilitação deve estar presente em pacientes comprometidos, a fim de que as complicações do implante possam ser rapidamente reconhecidas e tratadas quando necessário.

\section{REFERÊNCIAS}

1. AL YAFI F, et al. Is Digital Guided Implant Surgery Accurate and Reliable? Dental Clinics of North America, 2019; 63(3): 381-397.

2. ALBRECHT K, et al. The prevalence of dental implants and related factors in patients with Sjögren syndrome: Results from a cohort study. Journal of Rheumatology, 2016; 43(7): 1380-1385.

3. ASSOCIAÇÃO BRASILEIRA DE TRANSPLANTE DE ÓRGÃOS (ABTO). Registro Brasileiro de Transplantes (RBT) 2020. Dimensionamento dos Transplantes no Brasil e em cada estado (2013-2020). 2020. Disponível em: https://site.abto.org.br /wp- content/uploads/2021/ 03/rbt_2020_populaca 0-1-1.pdf. Acessado em: 09 de Julho de 2021.

4. BORNSTEIN MM, et al. Systemic conditions and treatments as risks for implant therapy. International Journal of Oral \& Maxillofacial Implants, 2009; 24(Suppl): 12-27.

5. BUSER D, et al. Modern implant dentistry based on osseointegration: 50 years of progress, current trends and open questions. Periodontology 2000, 2017; 73(1): 7-21.

6. MOLON RS, et al. Effect of the long-term administration of Cyclosporine A on bone healing around osseointegrated titanium implants: A histomorphometric study in the rabbit tibia. Microscopy Research and Technique, 2017; 80: 10001008.

7. DUTTENHOEFER F, et al. Dental implants in immunocompromised patients: a systematic review and meta-analysis. International Journal of Implant Dentistry, 2019; 5(1): 43

8. GU L. YU Y-C. Clinical outcome of dental implants placed in liver transplant recipients after 3 years: a case series. Transplantation Proceedings, 2011; 43(7): 2678-82.

9. GU L., et al.. Eleven dental implants placed in a liver transplantation patient: A case report and 5-year clinical evaluation. Chinese Medical Journal, 2011; 124(3): 472-5. 
10. HECKMANN SM, et al. Implant therapy following liver transplantation: Clinical and microbiological results after 10 years. Journal of Periodontology, 2004; 75: 909-913.

11. HOLT, CD. Overview of immunosuppressive therapy in solid organ transplantation. Anesthesiology Clinics, 2017; 35(3): 365-380.

12. KORFAGE A, et al. Dental Implants in patients with Sjögren'ssyndrome. Clinical Implant Dentistry and Related Research, 2016; 18(5): 937-945.

13. KOTSAKIS GA, et al. A systematic review of observational studies evaluating implant place- ment in the maxillary jaws of medically compromised patients. Clinical Implant Dentistry and Related Research, 2015; 17(3): 598-609.

14. MINISTÉRIO DA SAÚDE. Doação de órgãos: transplantes, lista de espera e como ser doador [internet]. Brasília: Ministério da Saúde; Brasil 2018. Disponível em: http://portalms.saude.gov.br/saude-de-a-z/doacao-de-orgaos. Acessado em: 09 de Julho de 2021.

15. MONTEBUGNOLI L, et al. Peri-Implant Response and Microflora in Organ Transplant Patients 1 Year after Prosthetic Loading: A Prospective Controlled Study. Clinical Implant Dentistry and Related Research, 2015; 17(5): $972-82$.

16. MONTEBUGNOLI L, et al. Bone response to submerged implants in organ transplant patients: a prospective controlled study. International Journal of Oral Maxillofacial Implants, 2012; 27(6): 1494-500.

17. NAKAGAWA A, et al. Implant treatment followed by living donor lung transplant: a follow-up case report. Journal of Prosthodontical Research, 2014; 58(2): 127-31.

18. PAREDES $\mathrm{V}$, et al. Implant treatment in pharmacologically immunosuppressed liver transplant patients: A prospectivecontrolled study. Clinical Oral Implants Research, 2017; 29(1): 28-35.

19. VISSINK A, et al. The medically compromised patient: Are dental implants a feasible option? Oral Diseases, 2018; 24(1-2): 253-26

20. ZHENG X, et al. Effect of FK-506 (tacrolimus) therapy on bone healing of titanium implants: a histometric and biomechanical study in mice. European Journal of Oral Sciences, 2017; 125(1):28-33. 